\title{
Exercise and Functional Capacity After Myocardial Infarction
}

\author{
Anne Folta, Bonnie L. Metzger
}

This paper summarizes the literature on the effects of both inpatient and outpatient exercise programs on functional capacity in patients categorized as having mild, moderate, severe and very severe left ventricular damage after myocardial infarction (MI). Current evidence suggests that inpatient activities of daily living programs do not improve functional capacity as measured by a predischarge exercise stress test. In contrast, outpatient exercise training programs can improve functional capacity through augmentation of skeletal muscle arteriovenous oxygen difference (oxygen extraction) in nearly all patients with mild left ventricular damage, in most patients with moderate damage and in a few patients with severe damage. The recently developed conceptual model of functional capacity in post-MI patients presented in this paper underscores the need for assessment of the level of structural and functional impairment of the left ventricle before the nurse prescribes any type of exercise program. It also enables the nurse to begin to explain why post-MI patients differ in their response to exercise. Further research is needed, however, before the model can be used for predicting exercise outcomes in the clinical setting.

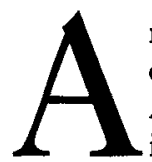

n estimated 900,000 Americans survive a myocardial infarction (MI) each year (American Heart Association, 1988). The ability of these patients to improve their functional capacity after exercise programs depends largely on the severity of the left ventricular pump damage (Carter \& Amundsen, 1977).

Functional capacity is defined conceptually as the product of cardiac output and skeletal muscle arteriovenous oxygen $\left(\mathrm{A}-\mathrm{V} \mathrm{O}_{2}\right.$ ) difference. Cardiac output (heart rate $\times$ stroke volume) reflects the ability of the left ventricle of the heart to pump oxygenated blood to the skeletal muscles; $\mathrm{A}-\mathrm{Vu} \mathrm{O}_{2}$ difference refers to the ability of the skeletal muscles to extract oxygen for energy production for use in contraction.

A frequently used, indirect measure of functional capacity is metabolic equivalent unit (MET). One MET is the patient's approximate oxygen consumption while sitting quietly in a chair and equals approximately $3.5 \mathrm{ml}$ of oxygen $/ \mathrm{kg} /$ min. Multiples of this measure are used to describe the oxygen consumption needed to perform a given workload. For example, exercise at the 2-MET level requires twice the oxygen consumption needed at rest $(7 \mathrm{ml} / \mathrm{kg} / \mathrm{min})$, the $3-\mathrm{MET}$ level requires three times the resting level $(10.5 \mathrm{ml} / \mathrm{kg} / \mathrm{min})$, and so on (Wilson, Fardy \& Froelicher, 1981). The MET level reached is calculated from speed and grade of the treadmill during a standardized exercise stress test before the occurrence of limiting signs and symptoms (e.g., exerciseinduced chest pain, dyspnea, ST-segment electrocardiographic [EKG] changes, life-threatening arrhythmias or profound muscular fatigue) (Kattus, 1975). An increase over time in the MET level reflects an improvement in functional capacity.

\section{Functional Capacity After Mild Left Ventricular Damage}

Patients categorized as having mild damage have sustained a small MI involving less than 10 percent of the left ventricular wall (see Table 1) and have an average ejection fraction of 0.58 units, which is normal or slightly reduced from normal levels $(E F=0.52-0.60$ units). Ejection fraction is a valid and reliable measure of the ability of the heart to pump blood and is defined as the ratio of stroke volume/end-diastolic volume. As illustrated in Table 1, the greater the level of damage, measured as infarct size, the more reduced is the ejection fraction. Borer et al. (1980) found that the ejection fraction did not change significantly from 48 hours to up to 1 year after the MI, suggesting that, once the left ventricular damage occurs, the resulting left ventricular damage is permanent and remains relatively stable over time. Ejection fraction alone differentiates effectively the levels of left ventricular damage. Other frequently used measures of the pumping ability of the heart that are less reliable are left ventricular end-diastolic pressure, stroke volume index (stroke volume/ body surface area) and cardiac index (cardiac output/body surface area).

Patients with mild left ventricular damage have a near normal left ventricular end-diastolic pressure and cardiac index, with a somewhat reduced stroke volume index. The noninfarcted segment of the heart compensates adequately for

ANNE FOLTA, R.N., Ph.D., Rho, is a research assistant, and BONNIE L. METZGER, R.N., Ph.D., Rho, is Associate Professor of Nursing, both at the University of Michigan School of Nursing, Ann Arbor. The authors acknowledge the support and assistance of Barbara A. Therrien, R.N., Ph.D., and R. Clinton Webb, Ph.D. Correspondence to Professor Metzger, 400 North Ingalls Bldg., Rm 2215, The University of Michigan, Ann Arbor, Ml 48109.

Accepted for publication June 7, 1989. 


\begin{tabular}{|c|c|c|c|c|}
\hline \multirow[b]{2}{*}{$\begin{array}{c}\text { Physiological } \\
\text { Measures } \\
(M \pm S D) \\
\end{array}$} & & & & \\
\hline & Mild & Moderate & Severe & Very Severe \\
\hline $\begin{array}{l}\text { Infarct size } \\
\quad \text { (percentage of left ventricular mass) } \\
\text { Ejection fraction }^{\mathrm{a}}\end{array}$ & $\begin{array}{c}\text { Small } \\
(<10 \%) \\
0.58 \pm .07\end{array}$ & $\begin{array}{l}\text { Medium } \\
(10-25 \%) \\
0.43 \pm 0.1\end{array}$ & $\begin{array}{c}\text { Large } \\
(-25 \%) \\
0.30 \pm .06\end{array}$ & $\begin{array}{c}\text { Very Large } \\
(>40 \%) \\
0.19 \pm .07\end{array}$ \\
\hline Normal $=0.52-0.60$ units & & & & \\
\hline $\begin{array}{l}\text { Left ventricular end-diastolic pressure } \\
\text { Normal }=12 \mathrm{mmHg}\end{array}$ & $15.8 \pm 3.3$ & $20 \pm 5.7$ & $26.7 \pm 6.3$ & $27.1 \pm 3.9$ \\
\hline $\begin{array}{l}\text { Stroke volume index } \\
\text { Normal }=54 \mathrm{ml} / \text { beat } / \mathrm{m}^{2}\end{array}$ & $42.2 \pm 8.7$ & $35.3 \pm 8.2$ & $29.5 \pm 5.5$ & $24.3 \pm 10.7$ \\
\hline $\begin{array}{l}\text { Cardiac index } \\
\quad \text { Normal }=2.9-3.3 \mathrm{~L} / \mathrm{min} / \mathrm{m}^{2}\end{array}$ & $2.7 \pm 0.5$ & $2.5 \pm 0.8$ & $2.2 \pm 0.9$ & $1.6 \pm 0.6$ \\
\hline $\begin{array}{l}\text { Heart rate } \\
\text { Normal }=60-100 \text { beats per minute }\end{array}$ & $73 \pm 14$ & $88.6 \pm 17.1$ & $89.1 \pm 9.6$ & $85.6 \pm 21$ \\
\hline $\begin{array}{l}\text { Mean arterial pressure } \\
\text { (diastolic }+1 / 3 \text { pulse pressure) } \\
\text { Normal }=90-105 \mathrm{mmHg}\end{array}$ & $103.5 \pm 24.2$ & $102 \pm 21$ & $106 \pm 21$ & $71 \pm 20$ \\
\hline $\begin{array}{l}\text { Adequacy of compensatory } \\
\text { mechanisms to maintain function }\end{array}$ & Very & Usually & Not Fully & Inadequate \\
\hline $\begin{array}{l}\text { Need for cardiac medications } \\
\text { to augment function }\end{array}$ & No & Sometimes & Usually & Always \\
\hline
\end{tabular}

aSignificantly differentiates levels from one another.

SOURCES: Forrester, Diamond \& Swan (1977); Rigaud et al. (1979); Rigo et al. (1974); Swan, Forrester, Diamond, Chatterjee \& Parmley (1972); Weber, Janicki, Russell \& Rackley (1978).

the small amount of left ventricular damage that has occurred in these patients, and medications that augment cardiac function are not needed. As a result, those with mild ventricular damage display no evidence of continuing cardiac ischemia, left ventricular failure, shock, significant cardiac arrhythmias, conduction disturbances or other serious illness after an MI (Swan et al., 1976).

When observed in the clinical setting, patients with mild left ventricular damage function without difficulty at rest, undergo inpatient activities of daily living (ADL) programs, and perform predischarge exercise stress testing without an ischemic response (occurrence of chest pain and/or STsegment changes on EKG). However, there are no published studies that evaluate whether or not inpatient ADL programs improve functional capacity in patients with mild left ventricular damage as currently measured by a low-intensity (4 MET) predischarge stress test during the first three to five days of hospitalization following the MI. An improvement in functional capacity probably would not be observed because the intensity and duration of ADL performed by the post-MI patient in the hospital are not sufficient to improve the A-V $\mathrm{O}_{2}$ difference (oxygen extraction) in skeletal muscle (Detry et al., 1971).

In contrast, a program of outpatient exercise training has been shown to increase functional capacity in patients with mild left ventricular damage. DeBusk, Houston, Haskell, Fry and Parker (1979) studied the effect of an 8-week outpatient home exercise training program on functional capacity begun at 3 weeks after $\mathrm{MI}$ in 12 male patients categorized as having mild left ventricular damage (see Table 2). The daily training program consisted of 30 minutes of stationary biking to maintain heart rate at 70 percent to 85 percent of the peak level obtained during exercise stress testing. Peak functional capacity in these patients 3 weeks after $\mathrm{MI}$ was $7.3 \pm 1.1$ METS (means \pm standard deviation) when tested using standard treadmill protocols. At the end of the exercise training program, 11 weeks after MI, the MET level increased to a mean of $10.3 \pm 1.4$, or a mean increase of $3.0 \pm 1.3$ METS. As expected, none of these patients had an ischemic response during exercise testing.

Dressendorfer, Scaff, Wagner and Gallup (1977) reported that three male patients, two to four years after an MI, underwent six months of training and were able to complete a marathon run (26.4 miles) at an average 10-MET pace for a five-hour period without the occurrence of an ischemic response. Patients with mild left ventricular damage can increase ejection fraction above resting values, through an increased stroke volume in response to exercise, although not to the same extent as can healthy adults. Peak functional capacity after mild left ventricular pump damage is reduced compared to that of healthy endurance athletes (16-20 METS) (Fox, Naughton \& Gorman, 1972).

\section{Functional Capacity After Moderate Left Ventricular Damage}

Patients categorized as having moderate left ventricular damage have sustained a medium sized infarction estimated to involve greater than 10 percent but less than 25 percent of the left ventricular wall. Average ejection fraction $(E F=$ 0.43 units) in this group was reduced significantly compared to patients with mild damage or to healthy adults (see Table 1). Many patients with moderate left ventricular damage have demonstrated early mild heart failure with accompanying mild pulmonary rales and a significantly elevated average left ventricular end-diastolic pressure of $20 \mathrm{mmHg}$. If ischemia, dyspnea, or hypotension occurs because of an inability to increase ejection fraction during exercise (Upton, Palmeri, Jones, Coleman \& Cobb, 1982), cardiac medication may be required to augment left ventricular function. 
Several nurses described the MET level that a sample of "stable" patients can reach after an MI without active exercise intervention at a given time (Halpenny \& Wills, 1977; Johnston, Watt \& Fletcher, 1981; Winslow, Lane \& Gaffney, 1985). Only Sivarajan et al. (1981) attempted to determine the effect of an inpatient ADL walking and calisthenic program (10.4 \pm 6.5 sessions) on functional capacity. Using a sample that consisted of 166 "stable" patients, they found no difference in ability to complete a low-intensity (4 MET) exercise stress test at discharge whether the patients were in the exercise group or part of the control group $(n=80)$ whose members only walked in the hall. The most likely explanation for the lack of an inpatient ADL effect in these patients is related to the low intensity and short duration of the program as well as the inability of a low-intensity stress test to differentiate between exercised and nonexercised patients. Thus, findings concerning the effect of low-intensity ADL programs on functional capacity immediately after an MI in patients with moderate left ventricular damage remain inconclusive. In the future, technical advances such as the ambulatory ventricular function monitor, which records simultaneously up to 3 hours of radionuclide and EKG data may be used to measure more effectively cardiac responses to inpatient ADL programs (Tamaki et al., 1987).

Studies conducted on patients with predominantly moderate left ventricular damage demonstrated that outpatient exercise training programs beginning at 3 weeks and ending 11 weeks after MI have a beneficial effect on functional capacity (see Table 2 ). The mean functional capacity rose significantly from $6.6 \pm 1.6$ to $11.0 \pm 1.6 \mathrm{METS}$, a total average increase of $4.4 \pm 1.7 \mathrm{METS}$, after completion of an 8 week outpatient exercise training program in 12 male patients who did not demonstrate an ischemic response during exercise testing (DeBusk et al., 1979). A smaller average increase in functional capacity (2.5 METS) was found by Eshani, Heath, Hagberg, Sobel and Holloszy (1981) when a one-year exercise training program was begun four months to five years after $\mathrm{MI}$ in 10 patients with moderate left ventricular damage without an ischemic response during stress testing. The above studies suggest that, to attain the maximal recovery of functional capacity after $\mathrm{MI}$, patients with moderate damage should begin their exercise training program

TABLE 2. Etfoct of Exerctse Training Programs on Functional Capacity by Level of Loft Ventrlcular Danage*

\begin{tabular}{lcccc}
$\begin{array}{l}\text { Level of Left } \\
\text { Ventricular } \\
\text { Damage }\end{array}$ & $\begin{array}{c}\text { Training } \\
\text { Time After } \\
\text { Infarction }\end{array}$ & $\begin{array}{c}\text { Pretraining } \\
M E T \text { Level } \\
(M \pm S D)\end{array}$ & $\begin{array}{c}\text { Posttraining } \\
\text { MET Level } \\
(M \pm S D)\end{array}$ & $\begin{array}{c}\text { Change in } \\
\text { METS } \\
(M \pm S D)\end{array}$ \\
\hline Mild & $\begin{array}{l}3 \text { to } 11 \\
\text { weeks }\end{array}$ & $7.3 \pm 1.1^{c}$ & $10.3 \pm 1.4$ & $+3.0 \pm 1.3$ \\
Moderate & $\begin{array}{l}3 \text { to } 11 \\
\text { weeks }\end{array}$ & $6.6 \pm 1.6$ & $11.0 \pm 1.6$ & $+4.4 \pm 1.7$ \\
Severe & $\begin{array}{l}3 \text { months } \\
\text { to } 1 \text { year }\end{array}$ & $7.0 \pm 1.9$ & $8.5 \pm 2.9$ & $+1.5 \pm 2.0$ \\
Very severe & - & - & - & - \\
\hline
\end{tabular}

in patients without an ischemic response during exercise testing.

${ }^{\mathrm{b}} \mathrm{MET}=$ metabolic equivalent unit which estimates oxygen consumption per $\mathrm{kg}$ of body weight. One MET equals $3.5 \mathrm{ml} / \mathrm{kg} / \mathrm{min}$.

'mean \pm standard deviation.

dprogram begun significantly later compared to those with mild and moderate damage.

exercise training contraindicated in this group. early at 3 weeks after MI while the infarction is healing and continue the program for at least 8 weeks.

Peak functional capacity was reduced significantly in 16 males who had an ischemic response during stress testing, as compared to those who did not (DeBusk et al., 1979). When ischemic responses occur, exercise programs are likely to be stopped temporarily or reduced in intensity so that the medication regimen can be changed. Those with frequent ischemic responses may be unable to complete an exercise training program.

Hung et al. (1984), who studied 53 men and Miller, Haskell, Berra and DeBusk (1984), who studied 27 patients, combined those with and without an ischemic response during exercise testing and reported only small increases (1.8 and 2.0 METS, respectively) in functional capacity after a 26week stationary bicycling protocol. But lower functional capacities associated with ischemic responses confounded the magnitude of the beneficial effect of the exercise training program. Findings based on studies that combine ischemic and nonischemic responses during exercise testing lose their value in predicting clinical outcomes.

Detry et al. (1971) suggested that the observed increases in functional capacity in post-MI patients following exercise training programs are related to an increased $\mathrm{A}-\mathrm{V} \mathrm{O}_{2}$ difference associated with an increase in both number and efficiency of mitochondria (cell energy sources) in trained skeletal muscle rather than to an increase in cardiac output. This augmentation of skeletal muscle function lowers indirectly the myocardial oxygen consumption, or work, of the heart by reducing the heart rate and systolic blood pressure needed to perform a given intensity of exercise, subsequently reducing the occurrence of angina and ST-segment EKG changes.

\section{Functional Capacity After Severe Left Ventricular Damage}

Patients with severe left ventricular damage have sustained an infarction of approximately 25 percent of the ventricular wall. Their average ejection fraction ( $E F=0.30$ units) is reduced significantly, as compared to patients with moderate left ventricular damage (see Table 1). Average left ventricular end-diastolic pressure was significantly elevated at 26.7 $\mathrm{mmHg}$ and stroke volume index and cardiac index were depressed markedly as compared to normal values. The heart is not able to compensate fully for the severe myocardial damage which has occurred, and as a result congestive heart failure is frequently observed. Ischemia or dyspnea is often observed in these patients at rest and is frequently observed during activities of daily living despite the use of cardiac medications. The majority of these patients cannot increase ejection fraction above resting levels during exercise, and have markedly increased peripheral vascular resistance, which increases the work of the heart (Vanhoutte, 1983).

The effect of inpatient ADL programs on functional capacity in patients with severe left ventricular damage has not been systematically studied. Starting inpatient ADL programs in these patients may be delayed because of the need for supplemental oxygen or reevaluation of the medication regimen. Also, predischarge exercise stress testing may be delayed because of the occurrence of symptoms of congestive heart failure. Typically, exercise other than the essential activities of daily living is begun at home after hospital discharge in patients with severe left ventricular damage. 
Supervised outpatient exercise training programs are begun significantly later in patients with severe damage, as compared to those with mild and moderate damage (see Table 2). The effect of an outpatient exercise training program begun 3 months after an MI was evaluated in 10 patients with severe damage, a mean ejection fraction less than 0.27 units (Conn, Williams \& Wallace, 1982). A special low-intensity, long-duration (9 month) walking, jogging and bicycling exercise training program increased significantly functional capacity from $7.0 \pm 1.9$ to $8.5 \pm 2.9$ METS. In 4 of the 10 patients studied, however, no increase in functional capacity was observed. Evidence suggests that, for patients with severe left ventricular damage to gain any benefit from exercise training, they must be willing to adhere to a long-term, low-intensity, supervised exercise training program.

\section{Exercise After Very Severe Left Ventricular Damage}

Patients with very severe damage have sustained a very large infarction involving about $\mathbf{4 0}$ percent of the left ventricular wall (see Table 1). Ejection fraction at rest and during movement are severely impaired $(E F=0.19$ units) and changes of body position alone may induce hypotensive episodes. Very severe myocardial damage is characterized by a greatly elevated left ventricular end-diastolic pressure and significantly reduced stroke volume index, cardiac index and mean arterial pressure (Forrester, Diamond \& Swan, 1977; Rigaud et al., 1979). These patients are maintained on cardiac inotropic and pressor agents (Swan et al., 1972) because compensatory mechanisms in the heart are inadequate and fail to improve left ventricular contractility. The mortality rate in patients with very severe damage is approximately 80 percent.

In patients with very severe left ventricular damage, the focus of care is on stabilization of blood pressure at rest. Therefore ADL programs during hospitalization are contraindicated unless the condition of the patient should improve dramatically. Exercise training programs and exercise stress testing are definitely contraindicated in these patients.

\section{Functional Capacity Conceptual Model}

A model of the relationships among the concepts of level of left ventricular pump damage, ability to perform exercise without an ischemic response, achievement of an exercise trained state and functional capacity (cardiac output $X$ skeletal muscle $\mathrm{A}-\mathrm{V} \mathrm{O}_{2}$ difference) is illustrated in Figure 1. The greater the level of left ventricular pump damage following an $\mathrm{MI}$, the less the heart is able to pump blood at rest and during exercise, as measured by ejection fraction or cardiac output. Further, the greater the level of pump damage present, the less the patient is able to perform exercise without an ischemic response during an inpatient or outpatient program, to reach an exercise trained state during an outpatient program and to improve functional capacity through an augmentation of skeletal muscle A-V $\mathrm{O}_{2}$ difference. Exercise training does not improve resting ejection fraction (Upton et al., 1982) or perfusion of blood in the left ventricular wall (Hung et al., 1984), and therefore probably does not directly improve cardiac function.

The nurse can augment skeletal muscle function during outpatient exercise training programs by assessing the level of left ventricular damage present, by prescribing an exercise intensity below the angina threshold and by helping patients monitor their own response to exercise. Current evidence suggests that outpatient exercise training programs, not lowintensity inpatient programs, can affect beneficially functional capacity in nearly all patients with mild left ventricular damage, in most patients with moderate ventricular damage without an ischemic response to stress testing and in a few selected patients with severe left ventricular damage. Improvement in functional capacity allows the patient to perform a higher intensity of work for a longer duration without an ischemic response and to increase the frequency of the workload performed.

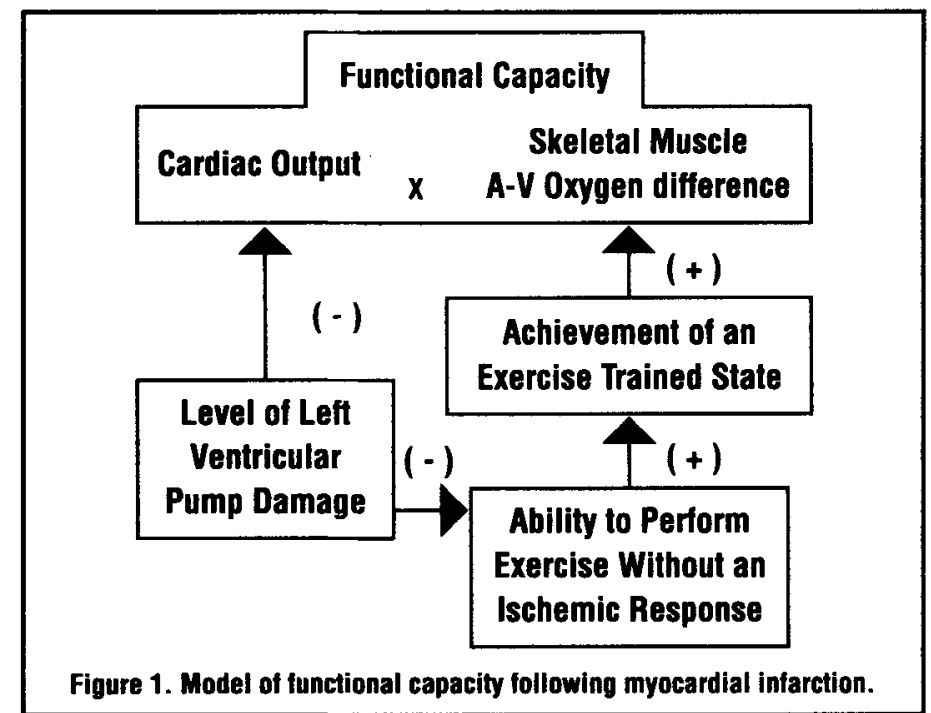

\section{Directions for Future Research}

Because of the paucity of research in this area and the complex nature and profound effect of the MI itself on the cardiovascular system, it is not surprising that exercise prescription guidelines for post-MI patients are not well developed (American College of Sports Medicine, 1986; American Nurses' Association, 1981). To separate the effects of the MI on cardiovascular function from the restorative effects of exercise training, the level of left ventricular damage should first be assessed. Norris, Caughey, Deeming, Mercer and Scott (1970) and Scheidt, Wilner, Fillmore, Shapiro and Killip (1973) developed indicies of left ventricular function that were based primarily on measures of left ventricular enddiastolic pressure following the MI, a much less reliable indicator of the pumping ability of the heart than ejection fraction. The levels of damage presented in this paper have an advantage over these classification systems since they are based primarily on the more reliable measure of ejection fraction (resting) but they are limited in generalizability because of the small sample sizes of the studies from which they are derived. Until technology develops to a point where ejection fraction can be measured during upright exercise, measures of ejection fraction at rest and during supine bicycling taken together with other measures probably provide the best estimate of level of left ventricular damage following an MI. Further research is needed, however, to assess the level of left ventricular damage in patients with multiple infarctions and right ventricular infarction. 
Male post-MI patients were used primarily in the studies reviewed. Further research on the effect of exercise programs in women would yield valuable information. Mickus (1986) began work in this area and found that 13 out of 25 female patients $(52 \%)$ had a reduced ability to resume normal activities of daily living after MI. Little work has been done, aimed specifically at examining the effect of an 8 week exercise training program on functional capacity in women following MI. Whether women require gender specific guidelines for exercise training and testing following $\mathrm{MI}$ is not known.

The ability of patients to improve their functional capacity after an MI is influenced significantly by their ability to exercise. Ideally, studies that control for both the level of left ventricular damage and gender are necessary to develop individualized exercise prescriptions for post-MI patients. We believe that the functional capacity model presented in this paper provides a framework for the development and conduct of these essential studies.

\section{References}

American College of Sports Medicine. (1986). Guidelines for exercise testing and training. Philadelphia: Lea and Febiger.

American Heart Association (1988). Heart facts. Dallas: AHA.

American Nurses' Association Division of Medical-Surgical Practice and American Heart Association Council on Cardiovascular Nursing. (1981). Standards of cardiovascular nursing practice. Kansas City: ANA

Borer, J., Rosing, D., Miller, R., Stark, R., Kent, K., Bacharach, S., Green, M. Lake, C., Cohen, H., Holmes, D.; Donohue, D., Baker, W., \& Epstein, S. (1980). Natural history of left ventricular function during 1 year after acute myocardial infarction: Comparison with clinical, electrocardiographic and biochemical determinations. American Journal of Cardiology, 46, 1-12.

Carter, C., \& Amundsen, L. (1977). Infarct size and exercise capacity after myocardial infarction. Journal of Applied Physiology, 42, 782-785

Conn, E., Williams, R., \& Wallace, A. (1982). Exercise responses before and after physical conditioning in patients with severely depressed left ventricular function American Journal of Cardiology, 49, 296-300.

DeBusk, R., Houston, N., Haskell, W., Fry, G., \& Parker, M. (1979). Exercise training soon after myocardial infarction. American Journal of Cardiology, 44, 1223-1229

Detry, J., Rousseau, M., Vandenbrouke, G., Kusumi, F., Brasseur, L., \& Bruce, R. (1971). Increased arteriovenous oxygen difference after physical training in coronary heart disease. Circulation, 44, 109-118.

Dressendorfer, R., Scaff, J., Wagner, J., \& Gallup, J. (1977). Metabolic adjustments to marathon running in coronary patients. Annals of the New York Academy of Sciences, 301, 466-483.

Eshani, A., Heath, G., Hagberg, J., Sobel, B., \& Holloszy, J. (1981). Effects of 12 months of intense exercise training ischemic ST-segment depression in patients with coronary artery disease. Circulation, 64, 1116-1123

Forrester, J., Diamond, G., \& Swan, H. (1977). Correlative classification of clinical and hemodynamic function after acute myocardial infarction. American Journal of Cardiology, 39, 137-145

Fox, S., Naughton, J., \& Gorman, P. (1972). Physical activity and cardiovascular health. III. The exercise prescription: Frequency and type of activity, Modern Concepts of Cardiovascular Disease, 41, 25-30.

Halpenny; C., \& Wills, R. (1977). Oxygen uptake and cardiovascular responses of myocardial infarction patients while dressing and walking on the treadmill. Communicating Nursing Research, 9, 135-145.

Hung, J., Gordon E., Houston, N., Haskell, W., Goris, M., \& DeBusk, R. (1984) Changes in rest and exercise myocardial perfusion and left ventricular function 3 to 26 weeks after clinically uncomplicated acute myocardial infarction: Effects of exercise training. American Journal of Cardiology, 54, 943-950.

Johnston, B., Watt, E., \& Fletcher, G. (1981). Oxygen consumption and hemodynamic and electrocardiologic responses to bathing in recent post-myocardial infarction patients. Heart \& Lung, 10, 666-670.

Kattus, A. (1975). Exercise testing and training of individuals with heart disease or at risk for its development. Dallas: AHA.

Mickus, D. (1986). Activities of daily living after myocardial infarction. Heart \& Lung, 15, 376-381.

Miller, N., Haskell, W., Berra, K., \& DeBusk, R. (1984). Home versus group exercise training for increasing functional capacity after myocardial infarction. Circulation, 70, 645-649
Norris, R., Caughey, D., Deeming, L., Mercer, C., \& Scott, P. (1970). Coronary prognostic index for predicting survival after recovery from acute myocardial infarction. Lancet, 2, 485-487.

Rigaud, M., Rocha, P., Boschat, J., Farcot, J., Bardet, J., \& Bourdarias, J. (1979). Regional left ventricular function assessed by contrast angiography in acute myocardial infarction. Circulation, 60, 130-139.

Rigo, P., Murray, M., Strauss, H., Taylor, D., Kelly, D., Weisfeldt, M., \& Pitt, B (1974). Left ventricular function in acute myocardial infarction evaluated by gated scintiphotography. Circulation, 50, 678-684

Scheidt, S., Wilner, G., Fillmore, S., Shapiro, M., and Killip, T. (1973). Objective haemodynamic assessment after acute myocardial infarction. British Heart Journal, 35, 908-916.

Sivarajan, E., Bruce, R., Almes, M., Green, B., Belanger, L., Lindskog, B., Newton, K., \& Mansfield, L. (1981). In-hospital exercise after myocardial infarction does not improve treadmill performance. New England Journal of Medicine, 305, 357-362.

Swan, H., Blackburn, H., DeSanctis, R., Frommer, P., Hurst, J., Paul, O., Rapaport, E., Wallace, A., \& Weinberg, S. (1976). Duration of hospitalization in "uncomplicated complete acute myocardial infarction". American Journal of Cardiology, 37, 413-418.

Swan, H., Forrester, J., Diamond, G., Chatterjee, K., \& Parmley, W. (1972) Hemodynamic spectrum of myocardial infarction and cardiogenic shock: A conceptual model. Circulation, 45, 1097-1110.

Tamaki, N., Gill, J., Moore, R., Yasuda, T., Boucher, C., \& Strauss, H. (1987). Cardiac responses to daily activities in normal subjects assessed by an ambulatory ventricular function monitor. American Journal of Cardiology, 59, 1164-1169.

Upton, M., Palmeri, S., Jones, R., Coleman, R., \& Cobb, F. (1982). Assessment of left ventricular function by resting and exercise radionuclide angiocardiography following acute myocardial infarction. American Heart Journal, 104, 1232-1243.

Vanhoutte, P. (1983). Adjustments in the peripheral circulation in chronic heart failure European Heart Journal, 4, 67-83.

Weber, K., Janicki, J., Russell, R., \& Rackley, C. (1978). Identification of high risk subsets of acute myocardial infarction. American Journal of Cardiology, 41, 197-203.

Wilson, P., Fardy, P., \& Froelicher, V. (1981). Cardiac rehabilitation, adult fitness, and exercise testing. Philadelphia: Lea \& Febiger.

Winslow, E., Lane, L., \& Gaffney, F. (1985). Oxygen uptake and cardiovascular responses in control adults and acute myocardial infarction patients during bathing. Nursing Research, 34, 164-169.

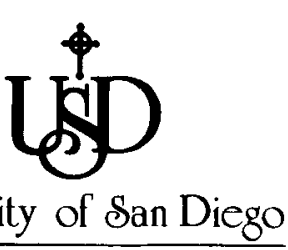

PHILIP Y. HAHN SCHOOL OF NURSINC THE UNIVERSITY OF SAN DIEGO Prestigious Private University Offers:

UNIQUE OPPORTUNITIES FOR

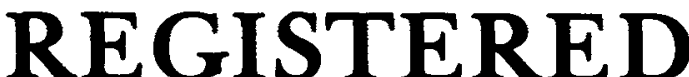
NURSES

- DOCTOR OF NURSING SCIENCE - MASTER OF SCIENCE IN NURSING - BACHELOR OF SCIENCE IN NURSING

BSN; MSN accredited National League for Nursing

Contact:

Philip Y. Hahn School of Nursing

Graduate Admissions or Undergraduate Admissions University of San Diego

Alcalá Park, San Diego, California $92110 \quad 619 / 260-4548$

The University of San Diego does nor discriminate on the basis of sex, race, color, religion, age, national origin, ancestry or handicap in its policies and programs. 